\title{
Distributed control system DCS using a PLC controller
}

\author{
Arkadiusz Hulewicz ${ }^{1, *}$, Zbigniew Krawiecki ${ }^{1}$, Krzysztof Dziarski ${ }^{1}$ \\ ${ }^{1}$ Poznan University of Technology, Institute of Electrical Engineering and Electronics, 3a Piotrowo street, 60965 Poznan, Poland
}

\begin{abstract}
The article presents The unconventional use of PLC controllers in a distributed control system DCS were presented in this article. The functionality, requirements and the costs of installation and operation of commercial DCS systems and those built on the basis of PLC logic controllers were analyzed. The features, the architecture and the structure of DCS systems based on PLC controllers have been described in details. The particular attention was paid to the allocation of the individual system components to the appropriate layers contained in their structure.
\end{abstract}

\section{Introduction}

The modern control systems and industrial facilities place ever higher demands related with their reliability. Commonly used centralized control requires the use of a central unit with high computing power and an extensive communication network. An alternative solution is the use of distributed control systems DCS.

Distributed Control System DCS is a combination of both hardware and software infrastructure ensuring automation of complex and distributed industrial processes. The basic idea of the DCS system is an integrated database that gathers all parameters related to the automated process. An important feature of this system is redundancy, which is based on deliberate redundancy of all components of the automation process. The DCS system can be realised in many ways, one of them is the system described in article, built only on the basis of a modular PLC controller.

Programmable Logic Controllers PLC are industrial computers used in controlling technological processes.. They enable simultaneous cooperation with many analog and digital signals, which guarantees their reliable operation in extended and distributed control systems, including DCS systems. [1,2]

The unconventional use of modular PLC controllers in a distributed DCS control system were presented in this article. The functionality of the proposed system was confirmed by the model of the production line built by the authors, controlled by means of modular PLC controllers connected in the DCS system. In addition, the basic issues related to the DCS system were discussed, the measurement, control and visualization elements used in this system were presented and the comparative analysis was presented in relation to the SCADA system and the PLC controller.

\section{Distributed DCS control system}

In modern automation, there is a tendency to replace traditional, centralized control systems DCS distributed systems based on the basic elements of automation, among which you can mention PLC controllers, industrial communication networks and visualization elements, and from the program side algorithms tested to work with complex systems. [3]

Using the MES (Manufacturing Execution System) application that manages production with optimization, material flow analysis, maintenance and flow of documentation and ERP (Enterprise Resource Planning) applications managing orders, finances and logistics, the DCS distributed control system is a bridge between technology processes and IT applications .

In the DCS system, controllers are connected to field devices and work computers by means of fast communication networks, whereas discrete devices with input and output modules via a communication bus. These devices communicate with PLC devices or other controllers using communication protocols among which one can mention Profibus, Modbus etc. [3]

\section{PLC controllers and DCS systems}

Modern control systems based on PLC controllers and SCADA software can effectively replace commercially available DCS systems. This adaptation is made possible by the parameters of currently available programmable logic controllers. [4,5]

Despite the increased functionality of PLC controllers and SCADA software, the systems based on them are still a centralized system. However, the use of distributed control does not require the use of better controllers, but rather linking them to a different network structure. Due to the above, according to the authors, the distributed control system can only be built using a PLC controller and a visualization system, which is illustrated

\footnotetext{
*Corresponding author: arkadiusz.hulewicz@put.poznan.pl
} 
in the model described in the article. In this model, in contrast to commercial DCS systems, PSI software was not used, which significantly increases DCS costs. The distributed systems are most often specialized to control a specific object, and the introduction of modifications is difficult. The system based on a PLC controller proposed in the article also provides redundancies, but in comparison to DCS systems it is more flexible and versatile. Also in terms of service, in the proposed system the role of the operator is supervision and response to failures. The DCS system requires a good knowledge of the controlled process and its configuration in online mode (it is possible to make changes in every aspect of control and visualization). [3] In addition, in these systems the process is often monitored by various components, including thermovision cameras. Using these cameras, their parameters and construction are important. [6]

\section{PLC and HMI panel in a distributed control system}

An alternative to the commercial distributed DCS control system is presented in the article the author's system using only a modular PLC controller and the HMI operator panel. [4,5] According to the accepted assumptions, this system should perform functions similar to the DCS system, but due to the program limitations it was impossible to complete all functions. The designed system was divided into the basic layers in which the following devices were assigned:

- TIA Portal V13 software to the engineering layer;

- two PLCs S7-1200 to the process layer;

- the HMI KTP 700 Basic panel to the operator layer;

- SCALANCE XB005 interface providing communication between all devices using Ethernet to the communication layer;

- embedded in the controller digital and analog inputs and outputs, the accuracy of which has been determined while maintaining certain guidelines to the input and output layer [7-9].

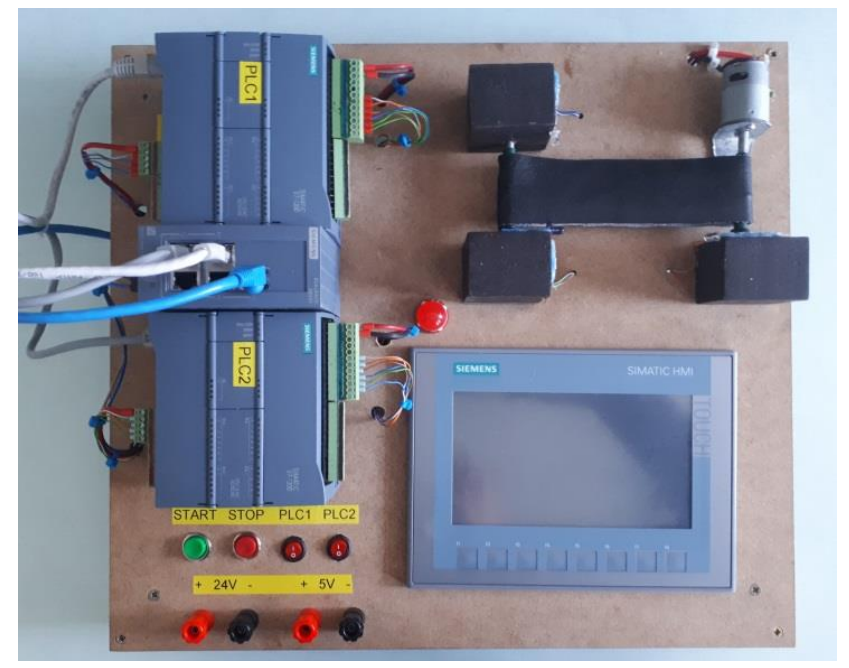

Fig. 1. The model of a distributed control system of a production line.
The proposed system (figure 1) is to control the model of the production line, and the redundancy was achieved thanks to the use of two Siemens S7-1200 controllers. The Master controller is PLC1 and its task is to control the process under typical operating conditions. The slave controller is a device marked as PLC2, and its task is to take over the control at the moment of PLC1 failure.

\section{Summary}

The issues related to the use of PLC controllers in distributed control systems were presented in this article. The built control model of a production line meets the set tasks, which in many aspects are similar to those carried out by commercial DCS systems. The proposed model can be effectively replaced by other devices installed in the real object. The disadvantage of the proposed solution is the fact that the software cooperating with the S7-1200 controllers prevents the definition of a common database for all variables. They are defined separately for each of the controllers.

The cost of implementing the control system is incomparably lower than the costs of implementing an analogous system using commercial DCS. Moreover, in the proposed solution, it is possible to freely choose the process control elements that can be independently modified.

Summing up, it can be stated that while designing distributed process control systems, only PLC controllers can be used, which with their availability and lower costs is the alternative solution.

\section{References}

1. E. A. Parr, Programmable controllers: an engineer's guide (Elsevier Ltd, Oxford, 2003)

2. W. Bolton, Programmable Logic Controllers (Elsevier Ltd, Oxford, 2009)

3. https://www.elprocus.com/distributed-controlsystem-features-and-elements/ 20.05.2019

4. https://support.industry.siemens.com/tedservices/D atasheetService/DatasheetService? format $=$ pdf\&mlf bs $=6$ ES7214-1 AG40-

0XB0\&language $=$ en \&caller $=$ SIOS $\quad 20.05 .2019$

5. https://support.industry.siemens.com/cs/pd/302298 ?pdti=td\&lc=en-PL 20.05.2019

6. K. Dziarski, J. Parzych, ITM Web Conf, 19, 1 (2018)

7. A. Hulewicz, Prz. Elektr, 11a, 319 (2010)

8. P. Otomanski, Z. Krawiecki, A. Odon, JPCS, 238, 012005 (2010).

9. A. Odon, Z. Krawiecki, Meas., 44, 8, 1406-1411, (2011) 